\title{
Urban Footprint Processor-Fully Automated Processing Chain Generating Settlement Masks From Global Data of the TanDEM-X Mission
}

\author{
T. Esch, M. Marconcini, Member, IEEE, A. Felbier, A. Roth, W. Heldens, M. Huber, \\ M. Schwinger, H. Taubenböck, A. Müller, and S. Dech, Member, IEEE
}

\begin{abstract}
The German TerraSAR-X add-on for Digital Elevation Measurement (TanDEM-X) mission (TDM) collects two global data sets of very high resolution (VHR) synthetic aperture radar (SAR) images between 2011 and 2013. Such imagery provides a unique information source for the identification of built-up areas in a so far unique spatial detail. This letter presents the novel implementation of a fully automated processing system for the delineation of human settlements worldwide based on the SAR data acquired in the context of the TDM. The proposed Urban Footprint Processor (UFP) includes three main processing stages dedicated to: i) the extraction of texture information suitable for highlighting regions characterized by highly structured and heterogeneous built-up areas; ii) the generation of a binary settlement layer (built-up, non-built-up) based on an unsupervised classification scheme accounting for both the original backscattering amplitude and the extracted texture; and iii) a final post-editing and mosaicking phase aimed at providing the final Urban Footprint (UF) product for arbitrary geographical regions. Experimental results assess the high potential of the TDM data and the proposed UFP to provide highly accurate geo-data for an improved global mapping of human settlements.
\end{abstract}

Index Terms-Automation, global mapping, settlements pattern, TerraSAR-X add-on for Digital Elevation Measurement (TanDEM-X), texture, urbanization.

\section{INTRODUCTION}

$\mathbf{U}$ RBANIZATION is one of the most pressing global challenges. More than $50 \%$ of the world's population is actually living in urban agglomerations and this proportion is expected to dynamically increase to $60 \%$ until 2030 and $70 \%$ until 2050 [1]. Accordingly, a profound understanding of the global spatial distribution and evolution of human settlements constitutes a key element in the current strategies to assure sustainable future development of urban and rural settlements [2]. To this aim, spaceborne Earth Observation (EO) has been established as an effective tool to provide global geoinformation on the location, spatial extent and distribution of urban areas. A comprehensive assessment of the currently available EO-based and EO-supported global human settlements

Manuscript received January 31, 2013; revised June 3, 2013; accepted July 5, 2013. Date of publication July 23, 2013; date of current version October 10, 2013

The authors are with the German Aerospace Center (DLR), Earth Observation Center, German Remote Sensing Data Center (DFD), 82234 Weßling, Germany (e-mail: thomas.esch@dlr.de; mattia.marconcini@dlr.de; andreas.felbier@dlr.de; achim.roth@dlr.de; wieke.heldens@dlr.de; martin. huber@dlr.de; max.schwinger@dlr.de; hannes.taubenboeck@dlr.de; andreas. mueller@dlr.de; stefan.dech@dlr.de).

Digital Object Identifier 10.1109/LGRS.2013.2272953 layers (GHSL) is given in [3] and [4]. In particular, both the reviews show that the existing GHSL are mainly derived from medium resolution (MR) optical EO data, hence exhibiting a comparably coarse spatial resolution (i.e., at least a few hundred meters). Nowadays, the MODIS 500 [5] and GlobCover 2009 [6] layers are considered to be the most accurate state-of-theart data sets provided on a global level. Nevertheless, their limited spatial resolution (i.e., 463 and $309 \mathrm{~m}$, respectively) do not allow an accurate characterization of rural and periurban areas, which are characterized by small and scattered villages and towns. Therefore, some recent initiatives aimed at improving the current GHSL by developing efficient processing techniques for delineating settlement extents based on high resolution (HR) and VHR EO data.

In [7], by processing and analyzing imagery of the Visible Infrared Imaging Radiometer Suite (VIIRS) on board of the Suomi NPP satellite, the intent is to provide by 2013 a new global nighttime lights product with significantly improved detection properties. An approach presented in [8], introduces an automatic algorithm for global built-up area recognition primarily based on HR/VHR optical imagery, but also capable of accounting for SAR data. Experimental results demonstrate the promising capabilities of the technique, although a global validation and the provision of a global product still owe. The integrated analysis of ASTER satellite images and GIS data is also used by [9] as a basis to provide a new GHSL.

Alternative approaches explicitly focus on the analysis of HR and VHR data collected by the latest generation of SAR satellites (i.e., Envisat-ASAR, ALOS-PALSAR, Radarsat-1/2, TerraSAR-X, TanDEM-X, COSMO-Skymed). In particular, with respect to optical sensors, the weather-independent, dayand-night data acquisition capability and the low sensitivity toward atmospheric effects of SAR systems make them particularly suitable to provide temporally and radiometrically consistent global data coverages. As an example, in [10] the capabilities of Envisat-ASAR data collected in Wide Swath Mode (at $75 \mathrm{~m}$ spatial resolution) are investigated to extract an urban settlement mask at $300 \mathrm{~m}$ spatial resolution that improves that derived from GlobCover 2009.

The authors yet explored and assessed the capabilities of the German TanDEM-X (TDM) mission [11] (whose primary goal is the production of a global digital elevation model-DEM-at $12 \mathrm{~m}$ spatial resolution) to monitor built-up areas using VHR imagery acquired by its two satellites TerraSAR-X (TSX) and TanDEM-X (TDX) [12]-[14]. However, to enable the mass 


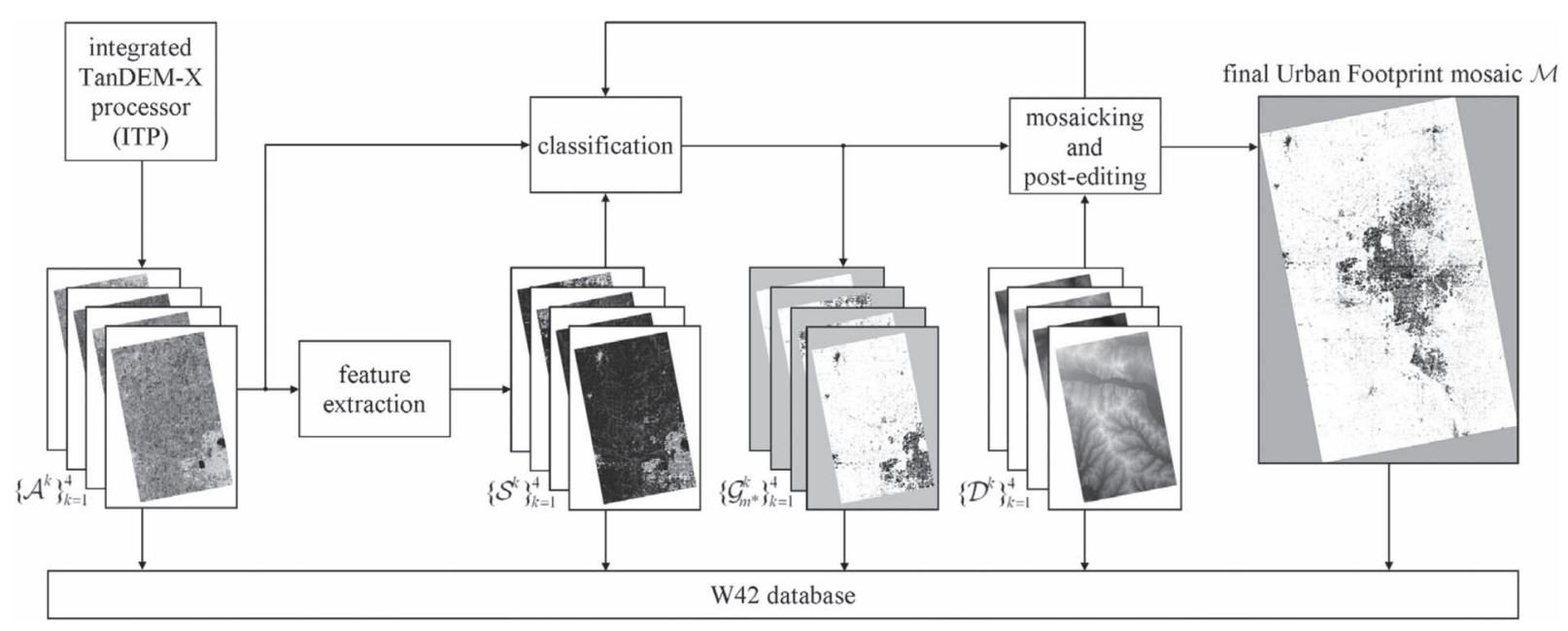

Fig. 1. Block scheme of the proposed UFP ( $\mathcal{A}$ denotes the geocoded backscattering amplitude, $\mathcal{S}$ the speckle divergence texture feature, $\mathcal{G}$ the produced binary UF map, and $\mathcal{D}$ the digital elevation model).

processing and analysis of a global data set of $\sim 180000$ complex SAR images collected in the first TDM global coverage, the basic methodology presented in [12]-[14] had, on the one hand, to be altered substantially in terms of the underlying analysis techniques. On the other hand, a novel technical framework orchestrating the entire suite of processing and analysis modules had to be developed in order to realize a fully automated, operational processing chain, namely the Urban Footprint Processor (UFP).

This letter introduces the conceptual implementation of the novel UFP framework along with an overview of the new or modified modules for feature extraction, classification and mosaicking and post-editing. The UFP has been implemented at the German Remote Sensing Data Center (DFD) of the German Aerospace Center (DLR) to generate a novel VHR GHSL at the unprecedented spatial resolution of $\sim 0.4$ arcseconds $(12 \mathrm{~m})$. Moreover, a public domain version downscaled at $\sim 3$ arcseconds (i.e., $\sim 50-75 \mathrm{~m}$ ) will be also produced.

In Section II, the methodological and technical framework of the UFP and its single modules are explained. In Section III, the binary settlement masks provided by the UFP are assessed and discussed for representative cases. Finally, conclusions and future developments are discussed in Section IV.

\section{URBAN FOOTPRINT PROCESSOR}

First methodological components for the identification of built-up areas from VHR TSX and TDX data acquired in Stripmap mode have been presented by the authors in [12][14]. However, to effectively handle a total number of $\sim 180000$ complex SAR images composing the first global TDM coverage (adding up to a final data volume of $\sim 300 \mathrm{~TB}$ ), the original modules and concepts had to be substituted or adapted to finally form a fully automated processing chain that is suitable for the production of a global product. In this context, a novel unsupervised classification method was implemented to automatically identify the optimal settings for the image classification of each specific investigated scene. For that purpose advanced Support Vector Data Description (SVDD) one-class classification [15] is employed. In addition, specific mosaicking and masking operations are applied in an automated post-editing phase to further improve the quality of the generated urban footprint (UF) product.

The UFP is implemented as a fully operational and datadriven system in C++ at DLR-DFD, where it is installed on a Sun X 4640 machine with eight CPUs at $2.6 \mathrm{GHz}$, six cores and 256 GB RAM. With that infrastructure an average of 400-500 images ( $\sim 1 \mathrm{~TB})$ can be analyzed and processed in $24 \mathrm{~h}$. A block scheme of the UFP is reported in Fig. 1. Inputs for the UFP are single look slant range complex (SSC) TSX/TDX images, acquired in Stripmap mode and delivered by the integrated TanDEM-X processor (ITP) [16]. The input images, as well as the intermediate layers and the final products are stored in a specific database (W42) which facilitates the effective handling and querying of the data [17].

In the following, we describe in detail the three main processing modules dedicated to: i) feature extraction; ii) classification; and iii) mosaicking and post-editing. Please note that we refer interchangeably to built-up or urban areas when referring to human settlements.

1) Feature Extraction: To derive effective texture information for highlighting regions characterized by highly structured and heterogeneous built-up areas we extract the so-called "speckle divergence" feature (as described in [13]), which accounts for the specific characteristics of SAR data that exhibit strong scattering due to double bounce effects in urban areas. In particular, focus is given to the analysis of the local speckle, whose development is estimated by means of the local image heterogeneity $\mathcal{H}$ (see [12]) defined as

$$
\mathcal{H}=\sigma_{\mathcal{A}} / \mu_{\mathcal{A}}
$$

where, for each pixel, $\mu_{\mathcal{A}}$ and $\sigma_{\mathcal{A}}$ represent the mean and the standard deviation of the original backscattering amplitude image $\mathcal{A}$, respectively, computed in a local neighborhood of $9 \times 9$ pixels. It is possible to prove that the following relationship exists between the image heterogeneity $\mathcal{H}$, the true image texture $\mathcal{T}$ and the fading texture $\mathcal{F}$ representing the heterogeneity caused by speckle [13]:

$$
\mathcal{H}^{2}=\mathcal{T}^{2} \mathcal{F}^{2}+\mathcal{T}^{2}+\mathcal{F}^{2}
$$


Textured surfaces (e.g., urban environments, woodlands) exhibit a significant amount of directional, non-Gaussian backscattering and are characterized by distinct structures, which lead to high $\mathcal{T}$, and hence to high values for $\mathcal{H}$. In turn, homogeneous surfaces without any true structuring (e.g., grasslands, non-cultivated bare soil) show almost no true texture $\mathcal{T}$, meaning that $\mathcal{H}$ approximates the fading texture $\mathcal{F}$ which results in very low values, which means that the backscattering is almost randomly distributed.

According to (2), the square of the local true image texture $\mathcal{T}^{2}$ (i.e., the speckle divergence $\mathcal{S}$ ) is obtained as

$$
\mathcal{T}^{2}=\mathcal{S}=\left(\mathcal{H}^{2}-\mathcal{F}^{2}\right)\left(1+\mathcal{F}^{2}\right)^{-1} .
$$

From the theory, $\mathcal{F}$ can be calculated as a function of the number of looks $N$ of a given SAR image. In particular, as done in [18], we approximated it as $\hat{\mathcal{F}}=0.5233 \cdot N^{-0.5}$. Since we considered radiometrically unenhanced SSC products, it holds that $N=N_{A} \cdot N_{R}$, where $N_{A}$ and $s N_{R}$ represent the number of looks in azimuth and range, respectively. In our case, being both $N_{A}=1$ and $N_{R}=1$ this led to a final constant value $\hat{\mathcal{F}}=0.5233[19]$.

2) Classification: The aim of the classification stage is to automatically derive a binary settlement layer (built-up, nonbuilt-up) for the investigated scene once provided as input with the backscattering image $\mathcal{A}$ and the corresponding speckle divergence $\mathcal{S}$. For this purpose we employed the fully automated and unsupervised technique whose main features are summarized in the following. It is worth noting that, to reduce the enormous amount of data to process, we preliminary applied the multi-looking technique implemented in the TerraSAR-X multi-mode SAR processor (TMSP). In particular, $\mathcal{A}$ and $\mathcal{S}$ are rescaled to a spatial resolution of about $\sim 0.4$ arcseconds (i.e., $\sim 12 \mathrm{~m}$ ), which is concurrently the highest resolution in which the global TDM DEM will be made available. This choice proved a reasonable tradeoff between noise reduction and fine detail preservation. Furthermore, a geocoding processor with a horizontal accuracy of up to $1 \mathrm{~m}$ [20] was also employed.

Pixels exhibiting high values for the speckle divergence are associated with built-up structures, whereas lower values correspond to non-urbanized area. Accordingly, the challenge is to identify for each specific investigated scene an optimal threshold for $\mathcal{S}$ that allows to effectively discriminate between urban and non-urban areas.

First, we directly mark as non-urban all the pixels whose backscatter amplitude is lower than the prefixed threshold $T h^{\mathcal{A}}=100$ (i.e., empirically derived from a number of experimental trials on hundreds of different images), as they are always associated with information classes not belonging to built-up areas (e.g., water bodies or surface types with a smooth meso-scale roughness).

Then, we identify a set of $M$ candidate thresholds for $\mathcal{S}$, $\left\{T h_{\mathrm{m}}^{\mathcal{S}}\right\}_{m=1}^{M}, T h_{1}^{\mathcal{S}}>\cdots>T h_{\mathrm{M}}^{\mathcal{S}}$, determined on the basis of the specific image dynamics. $\forall m$ samples are categorized into two different subsets, namely the urban $\left(\mathcal{U}_{m}\right)$ or non-urban $\left(\mathcal{L}_{m}\right)$ candidates depending on whether the corresponding speckle divergence value is greater or lower than $T h_{\mathrm{m}}^{\mathcal{S}}$, respectively. To this aim, a common choice in the literature is to analyze some specific information metrics, such as the Kullback-Leibler divergence $\left(D_{K L}\right)$ [21], which allows to measure the information lost when a distribution $Q$ is used to approximate a given distribution $P$. In particular, $D_{K L}$ is defined as

$$
D_{K L}[P \| Q]=\int_{-\infty}^{+\infty} p(\mathbf{x}) \cdot \ln [p(\mathbf{x}) / q(\mathbf{x})] d \mathbf{x}
$$

where $p(\mathbf{x})$ and $q(\mathbf{x})$ denote the probability density function (PDF) of $P$ and $Q$, respectively. The higher $D_{K L}$ the lower is the similarity between $P$ and $Q$. However, even if $D_{K L}$ is generally considered as a kind of a distance between two distributions, this is not formally true as it is not symmetric (i.e., $D_{K L}[P \| Q] \neq D_{K L}[Q \| P]$ ). Accordingly, we chose to employ the Jensen-Shannon divergence $\left(D_{J S}\right)$ [21], which is a symmetrized and smoothed version of $D_{K L}$ defined as

$$
D_{J S}[P \| Q]=\alpha \cdot D_{K L}[P \| M]+\beta \cdot D_{K L}[Q \| M]
$$

where $M=\alpha \cdot P+\beta \cdot Q$. In particular, we chose $\alpha=\beta=$ $1 / 2$ (referred to in the literature as specific $D_{J S}$ ) for which it holds that $D_{J S}[P \| Q] \in[0 ; \ln 2]$. In our case we fixed $P$ and $Q$ equal to $U_{m}$ and $L_{m}$, i.e., the probability distributions of $\mathcal{U}_{m}$ and $\mathcal{L}_{m}$, respectively.

High values for $T h_{m}^{\mathcal{S}}$ result in high values for $D_{J S}\left[U_{m} \| L_{m}\right]$, whereas as the threshold gets lower, also $D_{J S}\left[U_{m} \| L_{m}\right]$ decreases. It is possible to notice that, when the two distributions $U_{m}$ and $L_{m}$ start consistently overlapping, then there occurs a consistent fall in $D_{J S}\left[U_{m} \| L_{m}\right]$. When this occurs, the corresponding threshold $T h_{m^{*}}^{\mathcal{S}}$ is selected as optimal for the investigated image and the subset $\mathcal{U}_{m^{*}}$ is used to train a one-class classifier based on SVDD [15]. In particular, the SVDD technique aims at determining the hypersphere with minimum radius enclosing all the training samples available for the class of interest (i.e., the urban class in our case) and finally associates all the unknown samples falling inside the boundary with it. This approach allows increasing generalization and obtaining a more consistent and reliable final UF map $\mathcal{G}_{m^{*}}$.

3) Mosaicking and Post-Editing: Let us suppose that for a given study region $K$ different TDX images $\left\{\mathcal{I}^{k}\right\}_{k=1}^{K}$ are available. For all of them the corresponding optimal UF maps $\left\{\mathcal{G}_{m^{*}}^{k}\right\}_{k=1}^{K}$ are derived according to the method described above and then the final mosaic $\mathcal{M}$ is created. In the mosaicking process (which is straightforward being the maps geocoded) we compute the logical "or" between different UFs where they overlap (i.e., a pixel is associated to the urban class in the final mosaic if at least it is associated to the urban class in one of the UFs).

Generally, the proposed strategy for choosing the optimal threshold for the speckle divergence $\mathcal{S}$ proved to be very robust. However, sometimes it might occur that one or few images in the mosaic $\mathcal{M}$ exhibit slight overestimation or underestimation of urban areas with respect to neighboring scenes (hence resulting in a kind of striping effect). Such effects occurred, for instance, in case of images located at the coastline and mostly showing water with only a few land areas. This leads to extreme distributions which hinder the proper definition of accurate classification settings. To automatically detect and solve 
this issue we implemented a simple but effective approach. In particular, as each TDX image partly overlaps with all its neighbors, by comparing the number of samples classified as urban in the intersections it is possible to identify which maps are to be refined and whether overestimation or underestimation occurs. Being $\bar{k}$ the map to improve, in the former case we generate three additional versions $\left\{\mathcal{G}_{m *-i}^{\bar{k}}\right\}_{i=1}^{3}$ by choosing as many stricter thresholds for $\mathcal{S}$ and choose the one fitting better with its neighbors. Analogously, in the latter case we generate three additional maps $\left\{\mathcal{G}_{m^{*}+i}^{\bar{k}}\right\}_{i=1}^{3}$ by relaxing the thresholds for $\mathcal{S}$. The identified UF outlier tile is then substituted by the version which shows the lowest difference in the overlap area to its neighboring UF masks.

Finally, it might happen that, due to the effect of the topography on both the corresponding $\mathcal{A}$ and $\mathcal{S}$, highly mountainous areas, showing very high texture in combination with very high backscattering amplitude, are sometimes misclassified as built-up regions. To overcome this problem we implemented a dedicated mask derived from the analysis of the ASTER Global DEM [22]. In particular, we mark as non-urban all those pixels whose slope (intended as the maximum rate of height change between each pixel and its closest eight neighbors) is higher than 20 in the neighborhood of a local peak. Such strategy proved robust and allowed to minimize this type of error, while preserving at the same time urban settlements located on the side of the mountains.

\section{RESULTS}

To assess the accuracy of the proposed novel, fully automated UFP processing chain we compared the results obtained for five representative cases with those exhibited by the old semiautomated techniques presented in [12]-[14]. In particular, we considered a mosaic of 88 images $\left(\sim 90000 \mathrm{~km}^{2}\right)$ referring to the area of the megacity of New Delhi (IN) and four single images acquired over Buenos Aires (AR), Munich (DE), Nairobi (KE) and Padang (ID), respectively. Basis for this validation were UF masks in the public domain configuration at the spatial resolution of 0.4 arcseconds.

For the New Delhi test site a set of 2000 randomly distributed sample points (derived by blind photointerpretation of VHR optical images from GIS experts) is used as reference. Here, the overall accuracy (OA), user accuracy (UA), producer's accuracy (PA) and Kappa coefficient achieved based on the UFP processing came up to $86.6 \%, 92.7 \%, 76.7 \%$, and 0.73 , respectively. Some misclassification only occurred in villages characterized by very high density of trees (errors of omission) and rough, but small-scale topographic structures (e.g., dry river beds with high banks) or infrastructural elements such as railways or dikes along rivers and canals (errors of commission). Instead, using the old modules of the semi-automated approach, the OA, UA, PA, and Kappa coefficient took values of $55.1 \%$, $50.7 \%, 78.9 \%$, and 0.13 , respectively. This significantly weaker performance mainly arises from extensive over-classification in small-scale topographic structures which occurred in a significantly reduced amount when employing the UFP approach. Fig. 2 reports the original backscattering amplitude (a), the extracted speckle divergence (b), and the final UF mask (c). In

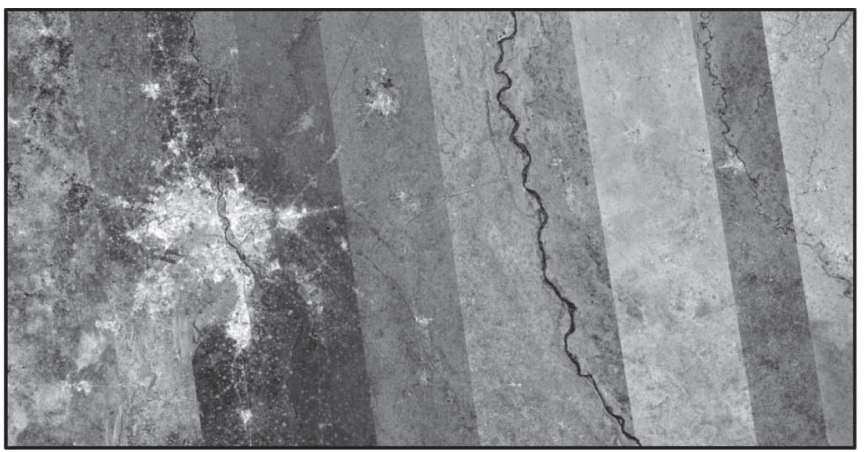

(a)

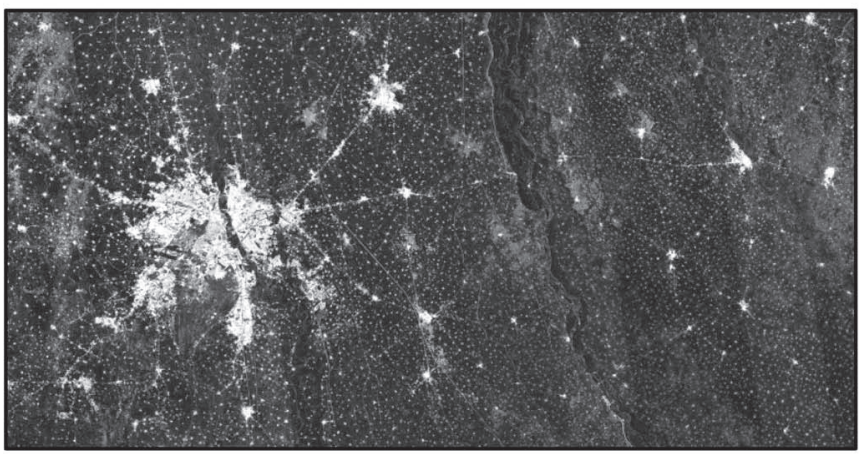

(b)

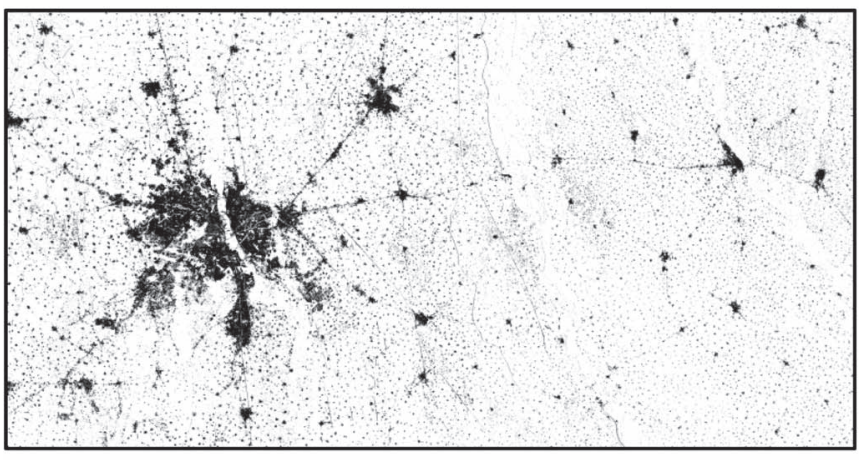

(c)

Fig. 2. Subset of the New Delhi test case (referring to an area of $\sim 250 \times$ $170 \mathrm{~km}$ including the megacity on the left) of the full mosaics generated from (a) the original backscattering amplitude images $\left\{\mathcal{A}^{k}\right\}_{k=1}^{88}$, (b) the extracted speckle divergence texture features $\left\{\mathcal{S}^{k}\right\}_{k=1}^{88}$, and (c) the final settlement masks $\left\{\mathcal{G}^{k}\right\}_{k=1}^{88}$ obtained with the presented UFP.

the amplitude mosaic, strong striping effects due to dissimilar acquisition conditions of different orbits are evident. This effect also occurs in the texture, although significantly extenuated. Nevertheless, the final mosaic obtained with the proposed UFP does not show any artifact of this type, hence confirming the high robustness and flexibility of the presented approach.

The results of the comparative analysis for the four single images are reported in Table I in terms of OA and Kappa. Reference points are the same as those considered in [12]. Here, it turns out that the quality is comparable for the cities Buenos Aires (BUE) and Munich (MUC), while the UFP procedure leads to significant improvements with respect to the old semiautomated approach for the cities of Nairobi (NBO) and Padang (PDG). The increased quality mainly results from the enhanced classification procedure. 
TABLE I

COMParative VALIDATION OF UF MASKS GENERATED By THE OLd Semi-Automated Approach and the NeW Fully Automated UFP For THE Cities of Buenos Aires (BUE), Munich (MUC), NAIROBI (NBO), AND PADANG (PDG)

\begin{tabular}{c|c|c|c|c}
\hline \hline \multirow{2}{*}{$\begin{array}{c}\text { Test } \\
\text { site }\end{array}$} & \multicolumn{2}{|c|}{ Semi-automated approach } & \multicolumn{2}{c}{ Fully-automated UFP } \\
\cline { 2 - 5 } & OA [\%] & Kappa & OA [\%] & Kappa \\
\hline BUE & 94.8 & 0.896 & 94.8 & 0.883 \\
MUC & 94.8 & 0.884 & 95.8 & 0.911 \\
NBO & 88.0 & 0.757 & 96.2 & 0.757 \\
PDG & 86.4 & 0.730 & 96.4 & 0.767 \\
\hline \hline
\end{tabular}

\section{CONCLUSION}

In this letter, we introduced the Urban Footprint Processor (UFP), a novel unsupervised and fully automated system developed at DLR for the global classification of urban areas using VHR SAR imagery from the TanDEM-X mission (TDM). The presented approach has been yet tested on thousands of images worldwide and preliminary validation results assess its great potential to support the research into global urbanization patterns and to investigate spatiotemporal aspects of urbanization and peri-urbanization. Extensive quantitative validation of the urban footprint (UF) products based on in situ ground truth will be the object of a future study.

The production of the first global UF layer from the first TDM data coverage ( $\sim 180000$ scenes covering $\sim 90 \%$ of the world land surface) is envisaged for 2014. In addition to the full-resolution $12 \mathrm{~m}$ product, also a public domain version downscaled at 3 arcseconds (i.e., $\sim 50-75 \mathrm{~m}$ ) will be generated.

Considering the challenges of a global UF production, we still investigate how to further improve the classification module of the UFP. To this aim we will systematically analyze the errors of omission and commission based on globally distributed VHR reference data sets. In this way we intend to identify potentially existing, systematic weaknesses of the UFP that might not have been discovered so far and at the same time gain a more precise knowledge on the tuning of the algorithms employed in the classification and mosaicking stage.

As potential future developments we also plan: i) to investigate the benefit which we expect might arise by considering the long-term coherence between the first (2011/2012) and second (2012/2013) TDM coverage in addition to the speckle divergence; and ii) to adapt the methodology to additional TSX/TDX imaging modes (e.g., ScanSAR) and other HR and VHR SAR sensors (e.g., Radarsat-2, ALOS-PALSAR, Sentinel-1). Moreover, TDM data provide further interesting perspectives for follow-on analyses - in particular, the extraction of building structures and the estimation of building densities based on texture measures as well as the modeling of building volume at block level by means of the TDM DEM.

\section{ACKNOWLEDGMENT}

The authors thank the TerraSAR-X and TanDEM-X Science Teams for providing the SAR data for this study.

\section{REFERENCES}

[1] World Population Prospects: The 2010 Revision, United Nations (01.12.12). [Online]. Available: http://esa.un.org/unpd/wpp/ Documentation/publications.htm

[2] D. J. Cieslewicz, "The environmental impacts of sprawl," in Urban Sprawl, Causes, Consequences \& Policy Responses, G. D. Squires, Ed. Washington, DC, USA: Urban Inst. Press, 2002, pp. 23-38.

[3] P. Gamba and M. Herold, Global Mapping of Human SettlementExperiences, Datasets, and Prospects. Boca Raton, FL, USA: CRC Press, 2009.

[4] D. Potere, A. Schneider, S. Angel, and D. L. Civco, "Mapping urban areas on a global scale: Which of the eight maps now available is more accurate?" Int. J. Remote Sens., vol. 30, no. 24, pp. 6531-6558, Dec. 2009.

[5] A. Schneider, M. A. Friedl, and D. Potere, "Mapping global urban areas using MODIS 500-m data: New methods and datasets based on urban ecoregions," Remote Sens. Environ., vol. 114, no. 8, pp. 1733-1746, Aug. 2010.

[6] GLOBCOVER 2009: Products description and validation report, Paris, France. [Online]. Available: http://due.esrin.esa.int/globcover/ LandCover2009/GLOBCOVER2009_Validation_Report_2.2.pdf

[7] Night Lights 2012, National Aeronautics and Space Administration (NASA) (31.01.2013). [Online]. Available: http://earthobservatory.nasa. gov/IOTD/view.php?id=79803

[8] M. Pesaresi, D. Ehrlich, I. Caravaggi, M. Kauffmann, and C. Louvrier, "Towards global automatic built-up area recognition using optical VHR imagery," IEEE J. Sel. Topics Appl. Earth Observ., vol. 4, no. 4, pp. 923 934, Dec. 2011.

[9] H. Miyazaki, X. Shao, K. Iwao, and R. Shibasaki, "An automated method for global urban area mapping by integrating ASTER satellite images and GIS data," IEEE J. Sel. Topics Appl. Earth Observ., vol. 6, no. 2, pp. 1004 1019, Apr. 2013.

[10] P. Gamba and G. Lisini, "A robust approach to global urban area extent extraction using ASAR Wide Swath Mode data," in Proc. TyWRRS, Naples, Italy, Sep. 12-14, 2012, pp. 1-5.

[11] G. Krieger, A. Moreira, H. Fiedler, I. Hajnsek, M. Werner, M. Younis, and M. Zink, "TanDEM-X: A satellite formation for high resolution SAR interferometry," IEEE Trans. Geosci. Remote Sens., vol. 45, no. 11, pp. 3317-3341, Nov. 2007.

[12] T. Esch, M. Thiel, A. Schenk, A. Roth, A. Müller, and S. Dech, "Delineation of urban footprints from TerraSAR-X data by analyzing speckle characteristics and intensity information," IEEE Trans. Geosci. Remote Sens., vol. 48, no. 2, pp. 905-916, Feb. 2010.

[13] T. Esch, A. Schenk, T. Ullmann, M. Thiel, A. Roth, and S. Dech, "Characterization of land cover types in TerraSAR-X images by combined analysis of speckle statistics and intensity information," IEEE Trans. Geosci. Remote Sens., vol. 49, no. 6, pp. 1911-1925, Jun. 2011.

[14] T. Esch, H. Taubenböck, A. Roth, W. Heldens, A. Felbier, M. Thiel, M. Schmidt, A. Müller, and S. Dech, "TanDEM-X mission-New perspectives for the inventory and monitoring of global settlement patterns," J. Appl. Remote Sens., vol. 6, no. 1, pp. 1-21, Oct. 2012.

[15] D. M. J. Tax and R. P. W. Duin, "Support vector data description," Mach. Learn., vol. 54, no. 1, pp. 45-66, Jan. 2004.

[16] T. Fritz, C. Rossi, N. Yague Martinez, M. Lachaise, and H. Breit, "Interferometric processing of TanDEM-X data," in Proc. IGARSS, Vancouver, BC, Canada, 2011, pp. 2428-2431.

[17] M. Habermeyer, U. Marschalk, and A. Roth, "W42-A scalable spatial database system for holding digital elevation models," in Proc. 17th Int. Conf. Geom., Fairfax, VA, USA, Aug. 12-14, 2009.

[18] Y. Huang and J. L. Genderen, "Evaluation of several speckle filtering techniques for ERS.1\&2 imagery," International Archives of Photogrammetry and Remote Sensing, vol. XXXI, pt. B2, pp. 164-169, 1996.

[19] E. Auquiere, SAR temporal series interpretation and backscattering modeling for maize growth monitoring. Louvain, Belgium: Presses Universitaires de Louvain, 2001.

[20] H. Breit, T. Fritz, U. Balss, M. Lachaise, A. Niedermeier, and M. Vonavka, "TerraSAR-X SAR processing and products," IEEE Trans. Geosci. Remote Sens., vol. 48, no. 2, pp. 727-740, Feb. 2010.

[21] J. Lin, "Divergence measures based on the Shannon entropy," IEEE Trans. Inf. Theory, vol. 37, no. 1, pp. 145-151, Jan. 1991.

[22] NASA: ASTER Global Digital Elevation Model Version 2, (31.01.2013). [Online]. Available: http://asterweb.jpl.nasa.gov/gdem.asp 